\title{
Chlamydia pneumoniae seroprevalence in immunocompetent and immunocompromised populations in Milan
}

\author{
F Blasi, R Cosentini, M Clerici Schoeller, A Lupo, L Allegra
}

\begin{abstract}
Background-Chlamydia pneumoniae is drawing increasing attention as an agent of respiratory tract infection. Specific antibody prevalence in western countries is low in preschool children and reaches more than $50 \%$ in adults. However, little is known about the prevalence of this infection in immunocompromised subjects such as HIV-I infected patients. The aim of this study was to evaluate the seroprevalence of $\mathrm{Chl}$ pneumoniae in immunocompetent and immunocompromised (HIV-I infected) paediatric and adult populations.
\end{abstract}

Methods-Between March 1991 and September 1992764 healthy subjects (421 men and 343 women, age range six months-81 years), 96 HIV-I infected (73 men and 23 women, age range 18-35 years) and 126 HIV-I negative intravenous drug users (92 men and 34 women, age range 18-37 years), and 50 children ( 23 boys and 27 girls, age range 8-123 months) with vertically transmitted HIV-I infection were studied. For each subject an HIV-I test (ELISA and Western blot) was performed, together with a microimmunofluorescence test for IgG and IgM antibodies to $C h l$ pneumoniae specific antigen (TW-183).

Results-In the healthy population a low prevalence $(11 \%)$ was observed in children under 10 years of age, which increased progressively to $58 \%$ in adults over 70 years. In the HIV-I infected population Chl pneumoniae seroprevalence was higher than in immunocompetent controls (children, 26\% v 11\%; drug users, $60 \% v 40 \%$ ). Moreover, in drug users this difference was also observed in comparison with HIV-I negative intravenous drug users $(60 \% v 33 \%)$.

Conclusions-Our data on Chl pneumoniae seroprevalence in a healthy population are consistent with those reported by others in western countries. Moreover, HIV-I infected subjects seem to be at higher risk of developing Chl pneumoniae infections.

(Thorax 1993;48:1261-1263)

Chlamydia pneumoniae is a recently recog- nised cause of respiratory tract infections. ${ }^{12}$ It has been classified as a third species of the Chlamydia genus by means of ultrastructural and DNA homology analysis. ${ }^{3}$ It is an obligate intracellular, Gram negative bacterium involved in a wide spectrum of respiratory tract infections; in fact, this agent can cause both upper respiratory tract infection (pharyngitis, sinusitis, and otitis) and low respiratory tract infection (acute bronchitis, exacerbations of chronic bronchitis, and community acquired and nosocomial pneumonias)..$^{4-6}$ Several studies have recently stressed its importance in the development of respiratory disease, showing a high incidence and prevalence of infections worldwide.

Specific antibody prevalence in western countries is low in preschool children and reaches more than $50 \%$ in adults, remaining high in old age because of Chl pneumoniae reinfection among adults. ${ }^{7}$ Little is known, however, about the prevalence of this infection in immunocompromised subjects such as HIV-I infected patients. Two recent papers reported evidence of $C h l$ pneumoniae infection in HIV-I patients with pulmonary disease. ${ }^{89}$

The aim of the present study was to evaluate Chl pneumoniae seroprevalence in immunocompetent and immunocompromised (HIV-I infected) paediatric and adult populations in Milan.

\section{Methods}

Serum samples were obtained from 764 (421 men and 343 women, age range six months-81 years) asymptomatic blood donors, subjects participating in a programme for atherosclerosis primary prevention, and children screened for hepatitis B infection. Serum samples were also obtained from a large group of intravenous drug users, 96 of whom were asymptomatic but HIV-I positive (73 men, age range 18-35 years, mean (SD) $29 \cdot 1(5 \cdot 2))$, and 126 who were HIV-I negative (92 men, age range 18-37 years, mean (SD) $29.6(5 \cdot 8))$. We also studied 50 children (23 boys, age range 8-123 months, mean (SD) 48 (10)) with vertically transmitted HIV-I infection.

All serum samples were obtained between March 1991 and September 1992. Informed consent to participate in the study was obtained from all subjects (parents for children). 


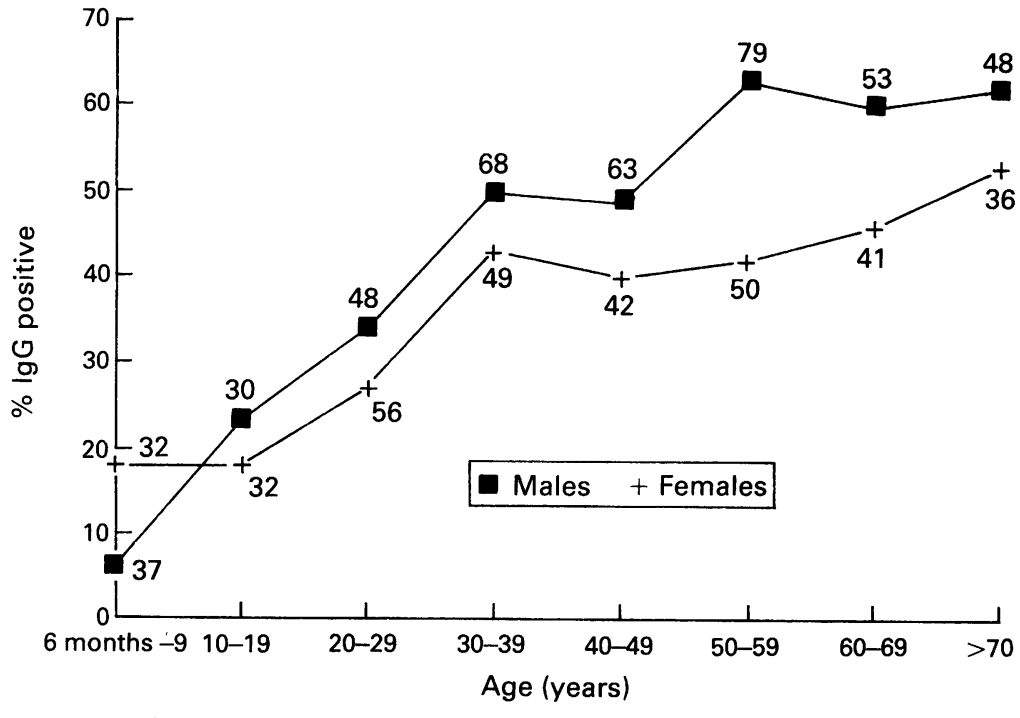

Percentage of positive results in serological tests for IgG to Chlamydia pneumoniae in healthy subjects according to age and sex. Numerical values indicate numbers of subjects.

The study was approved by the ethical committee of the University of Milan. We performed an HIV-I test (ELISA and Western blot) together with a microimmunofluorescence test $^{10}$ for IgG and IgM antibodies to $C h l$ pneumoriae specific antigen (TW-183) prepared by the Washington Research Foundation, Seattle, USA, on each subject. Microimmunofluorescence results were classified as previously reported ${ }^{2}$ : threshold titre positivity for IgM and IgG were $>1: 16$ and $>1: 64$, respectively.

\section{STATISTICAL METHODS}

Comparison of seroprevalence of $\mathrm{Chl}$ pneumoniae was performed with Fisher's exact test or $\chi^{2}$ test.

\section{Results}

The seroprevalence of Chl pneumoniae in the healthy population is shown in the figure. There was a low prevalence $(11 \%)$ in normal children under 10 years of age which increased progressively to $58 \%$ in adults over 70 years. Males showed a higher prevalence at all ages with the exception of children under 10 years $(-12 \%)$. The greatest difference $(+21 \%)$ was observed in subjects between 50 and 59 years. The seroprevalence of Chl pneumoniae in the HIV-I infected intra-

Table 1 Demographic characteristics and serological data in HIV-I positive and HIV-I negative intravenous drug users and in control subjects matched for age and sex

\begin{tabular}{llll}
\hline & \multicolumn{2}{l}{ Intravenous drug users } & \\
\cline { 2 - 3 } & $\begin{array}{l}\text { HIV-I positive } \\
(96)\end{array}$ & $\begin{array}{l}\text { HIV-I negative } \\
(126)\end{array}$ & $\begin{array}{l}\text { Controls } \\
(147)\end{array}$ \\
\hline M/F & $73 / 23$ & $92 / 34$ & $95 / 52$ \\
Mean age (years) & $29 \cdot 1$ & $29 \cdot 6$ & $28 \cdot 5$ \\
Age range (years) & $18-35$ & $18-37$ & $18-34$ \\
IgG $\geqslant 1: 64$ & $58(60 \%)^{\star}$ & $41(33 \%)$ & $59(40 \%)$ \\
IgM $\geqslant 1: 16$ & $4(4 \%)$ & 0 & 0 \\
\hline
\end{tabular}

${ }^{\star} \mathrm{p}<0.01$ Chlamydia pneumoniae seroprevalence in HIV-I positive $v$ HIV-I negative intravenous drug users $v$ controls $\left(\chi^{2}\right.$ test).
Table 2 Demographic characteristics and serological data of HIV-I vertically infected children and control subjects matched for age and sex

\begin{tabular}{|c|c|c|}
\hline & $\begin{array}{l}\text { HIV-I positive } \\
\text { (50) }\end{array}$ & $\begin{array}{l}\text { Controls } \\
\text { (87) }\end{array}$ \\
\hline $\begin{array}{l}M / F \\
\text { Age range (months) } \\
I g G \geqslant 1: 64 \\
I g M \geqslant 1: 16\end{array}$ & $\begin{array}{l}23 / 27 \\
8-123 \\
13(26 \%)^{\star} \\
2(4 \%)\end{array}$ & $\begin{array}{l}42 / 45 \\
6-120 \\
9(11 \%) \\
0\end{array}$ \\
\hline
\end{tabular}

venous drug users was significantly higher ( $\mathrm{p}<0.01, \chi^{2}$ test) than both HIV-I negative $\overrightarrow{0}$ intravenous drug users and immunocompe- $\overrightarrow{-}$ tent subjects matched for age and sex (table $\vec{\omega}$ 1). Four (4\%) HIV-I positive intravenous drug users had an IgM titre $>1: 16$, suggesting acute infection. Children with vertically $\stackrel{+}{\infty}$ infected HIV-I had a significantly higher $\vec{N}$ prevalence ( $p<0.05$, Fisher's exact test) than $\vec{N}$ healthy controls matched for age and sex 9 (table 2). Two (4\%) HIV-I positive children 음 also had an IgM titre $>1: 16$, suggesting acute infection.

\section{Discussion}

Chlamydia pneumoniae is an emerging respira- $\vec{\bullet}$ tory pathogen worldwide, causing more than $10 \%$ of community acquired pneumonias with a high seroprevalence in the adult population. ${ }^{7}$ Moreover, a possible role for this agent in low respiratory tract infections in immunocompromised patients has been sug- $\frac{\circ}{\Phi}$ gested. ${ }^{89}$ To our knowledge, no data on seroprevalence in HIV-I infected subjects have응 been reported in the literature and the epi- $\frac{3}{2}$ demiology of Chl pneumoniae in Italy is unknown.

We therefore studied $C h l$ pneumoniae sero-ô prevalence in a sample of the population of $\tilde{x}_{x}$ Milan, and in two groups of immuno- $-\dot{-}$ compromised subjects represented by HIV-I infected drug users and HIV-I verticallyô
infected children.

The microimmunofluorescence serological 윽 test used in this study, although quite time $>$ consuming, is a specific and sensitive diagnostic method for Chl pneumoniae infection. N Thom et $\mathrm{al}^{4}$ reported that isolation of $\mathrm{Chl}_{N}$ pneumoniae without serological evidence of acute infection is rare and occurred in only ${ }^{\omega}$ $4 / 1100$ of their patients. Grayston ${ }^{11}$ in a large $e^{2}$ study involving more than 6000 subjects confirmed that non-specific cross reactions in the microimmunofluorescence test between $C h l_{\square}$ trachomatis and Chl pneumoniae do not occuro when the test is interpreted properly for specific reactions.

Our data on population seroprevalence in the healthy population are consistent with those reported by Grayston $e t a l^{7}$ and showo that infection is endemic in the general adult population in our area, with a similar pattern to that in other western countries. Chl pneumoniae seroprevalence in HIV-I infected subjects was significantly higher than in healthy subjects for both HIV-I vertically infected 
children and HIV-I infected intravenous drug users. Interestingly, in the latter group seroprevalence was also significantly higher when compared with a group of HIV-I negative intravenous drug users, suggesting that the risk factor for Chl pneumoniae infection is immunodeficiency rather than life style.

The high seroprevalence of Chl pneumoniae in HIV-I infected subjects, and the casual finding of antibody titre suggesting acute infection, together with the recent reports of clinical Chl pneumoniae infections in immunocompromised subjects ${ }^{89}$ confirm the potential role for this agent in the pathogenesis of respiratory tract infections in HIV-I infected subjects.

Further studies are needed to elucidate fully the pathogenetic role of $\mathrm{Chl}$ pneumoniae in HIV-I infected subjects, because this high antibody prevalence could be the result of either a greater rate of infection in immunocompromised subjects or a polyclonal immunoglobulin activation commonly found in HIV patients.

This study was supported by the National Research Council (CNR) targeted project "Prevention and control of disease factors", PF 41.
1 Grayston JT, Kuo CC, Wang SP, Altman J. A new Chlamydia psittaci strain, TWAR, isolated in acute respiratory tract infections. $N$ Engl $\mathcal{F}$ Med 1986;315:161-8.

2 Thom DH, Grayston JT, Wang SP. Chlamydia pneumoniae strain TWAR, Mycoplasma pneumoniae and viral infection in acute respiratory disease in a university student health clinic population. Am $\mathcal{f}$ Epidemiol 1990; 161:248-56.

3 Grayston JT, Kuo CC, Campbell LA, Wang SP. Chlamydia pneumoniae sp.nov. for Chlamydia sp. strain TWAR. Int ₹ Syst Bacteriol 1989;39:88-90.

4 Thom DH, Grayston JT. Infections with Chlamydia pneumoniae strain TWAR. Clin Chest Med 1991;12:245-56.

5 Blasi F, Legnani D, Lombardo VM, Negretto GG, Magliano E, Pozzoli $\mathrm{R}$, et al. Chlamydia pneumoniae infection in acute exacerbations of COPD. Eur Respir $\mathcal{F}$ 1993;6:19-22.

6 Almirall J, Moratò I, Riera F, Verdaguer A, Priu R, Coll $\mathrm{P}$, et al. Incidence of community-acquired pneumonias and occurrence of Chlamydia pneumoniae infection: a prospective multicentre study. Eur Respir F 1993;6:14-8.

7 Grayston JT, Campbell LA, Kuo CC, Mordhorst CH, Saikku P, Thom DH, et al. A new respiratory tract pathogen: Chlamydia pmeumoniae strain TWAR. F Infect Dis 1990;161:618-25.

8 Augenbraun MH, Roblin MR, Chirwing K, Landman D, Hammerschlag MR. Isolation of Chlamydia pneumoniae from lungs of patients Infected with the human immunodeficiency virus. ff Clin Microbiol 1991;29: 401-2.

9 Clark R, Mushatt D, Fazal B. Case report: Chlamydia pneumoniae pneumonia in an HIV-infected man. Am $\mathcal{F}$ Med Sci 1991;302:155-6.

10 Wang SP, Grayston JT. Immunologic relationship between genital TRIC, lymphogranuloma venereum, and related organisms in a new microtiter indirect immunofluorescence test. Am $\mathcal{f}$ Ophthalmol 1970;70: immunofict

11 Grayston JT. Chlamydia pneumoniae, strain TWAR, a new important pathogen. In: Vaheri A, Tilton RC, Balows A, eds. Rapid methods and automation in microbiology and immunology. Berlin: Springer-Verlag, 1991:284-98. 